\title{
Quality of reduction correlates with clinical outcome in pelvic ring fractures
}

\author{
Pastor, Torsten ; Tiziani, Simon ; Kasper, Cla Duri ; Pape, Hans-Christoph ; Osterhoff, Georg
}

\begin{abstract}
INTRODUCTION Posttraumatic pelvic deformity is associated with pain and loss of function. This study aimed to test the correlation of functional outcome in patients with pelvic fractures with the postoperative radiographic quality of reduction. METHODS Consecutive patients with an isolated traumatic pelvic fracture that required closed or open reduction between 07/2015 and 07/2017 and had a completed follow-up of at least 6 months were included $(\mathrm{N}=31$, mean age 50 years, SD 21 years, range, 16 to 88 years). Majeed and Timed Up Go were obtained from a prospective outcome database at 6 months and last follow-up (mean 10 months, SD 5 months). Quality of pelvic ring reduction was determined on postoperative radiographs as described by Matta, Sagi and Keshishyan/Lefaivre. RESULTS Clinical outcome at 6 months as measured by the Majeed and the Timed Up Go correlated moderately with Keshishyan/Lefaivre's pelvic asymmetry value (Pearson R: -0.520 and $0.585, \mathrm{p} \quad 0.003$ ) and the pelvic deformity index $(-0.527$ and $0.503, \mathrm{p} \quad 0.004)$. There was a weak correlation between the Timed Up Go and the radiographic grading system as described by Matta/Tournetta at 6 months $(0.408, \mathrm{p}=0.023)$ and at last follow-up $(0.380, \mathrm{p}=0.035)$. CONCLUSIONS This study showed a moderate correlation of the clinical outcome at 6 months with postoperative quality of radiographic reduction when measured with the method described by Keshishyan and Lefaivre. Although having only descriptive value due to the small cohort, our findings underline the importance of anatomic reduction and restoration of pelvic symmetry in patients with pelvic trauma. Future studies with more patients and more investigators are required and reliability and validity of functional outcome scores needs to be further assessed to predict outcome in patient with fractures of the pelvic ring. LEVEL OF EVIDENCE Level IV (case series).
\end{abstract}

DOI: https://doi.org/10.1016/j.injury.2019.04.015

Posted at the Zurich Open Repository and Archive, University of Zurich

ZORA URL: https://doi.org/10.5167/uzh-170680

Journal Article

Accepted Version

Originally published at:

Pastor, Torsten; Tiziani, Simon; Kasper, Cla Duri; Pape, Hans-Christoph; Osterhoff, Georg (2019). Quality of reduction correlates with clinical outcome in pelvic ring fractures. Injury, 50(6):1223-1226.

DOI: https://doi.org/10.1016/j.injury.2019.04.015 
2

\section{Quality of reduction correlates with clinical outcome} in pelvic ring fractures

6 


\section{Abstract}

Introduction Posttraumatic pelvic deformity is associated with pain and disabilityloss of function.

This study aimed to test the correlation of functional outcome in patients with pelvic fractures with the postoperative radiographic quality of reduction.

Methods Consecutive patients with an isolated traumatic pelvic fracture that required closed or open reduction between 07/2015 and 07/2017 and had a completed follow-up of at least 6 months were included ( $\mathrm{N}=31$, mean age 50 years, SD 21 years, range, 16 to 88 years). Majeed and Timed Up \& Go were obtained from a prospective outcome database at 6 months and last follow-up (mean 10 months, SD 5 months). Quality of pelvic ring reduction was determined on postoperative radiographs as described by Matta, Sagi and Keshishyan/Lefaivre.

Results Clinical outcome at 6 months as measured by the Majeed and the Timed Up \& Go correlated moderately with Keshishyan/Lefaivre's pelvic asymmetry value (Pearson R: -0.520 and 0.585 , $\mathrm{p} \leq 0.003)$ and the pelvic deformity index $(-0.527$ and $0.503, \mathrm{p} \leq 0.004)$. There was a weak correlation between the Timed Up \& Go and the radiographic grading system as described by Matta/Tournetta at 6 months $(0.408, \mathrm{p}=0.023)$ and at last follow-up $(0.380, \mathrm{p}=0.035)$.

Conclusions This study showed a moderate correlation of the clinical outcome at 6 months with postoperative quality of radiographic reduction when measured with the method described by Keshishyan and Lefaivre. Although having only descriptive value due to the small cohort, our findings underline the importance of anatomic reduction and restoration of pelvic symmetry in patients with pelvic trauma. Future studies with more patients and more investigators are required and reliability and validity of functional outcome scores needs to be further assessed to predict outcome in patient with fractures of the pelvic ring.

\section{Level of evidence: Level IV (case series)}

Key words: pelvic ring fractures, pelvis fracture, outcome, pelvic deformity. 


\section{Introduction}

Pelvic ring injuries are associated with high-energy trauma and high mortality due to critical blood loss and concomitant injuries ${ }^{1,2}$. In those who survive, persistent pelvic ring deformity can lead to pain and limitation in activities of life. It has been suggested that improved reduction correlates with better functional outcome and that anatomic reduction of the pelvic ring is as important as simple stabilization ${ }^{3}$. Radiographic outcome still represents the most commonly reported outcome in studies on pelvic ring fractures ${ }^{4}$. There exist several techniques to measure pelvic deformity on radiographs ${ }^{5-}$ ${ }^{7}$. The literature on the clinical validity of these techniques, however, is scarce.

In a systematic review, Lefaivre et al ${ }^{4}$ identified only two radiographic measurement methods that used a clear and reproducible measurement technique on postoperative x-rays with acceptable interobersever reliabilities for describing radiographic outcomes: the pelvic ratio method described by Sagi et al. ${ }^{7}$ and the cross-measurement technique initially described by Keshishyan and modified by Lefaivre et al ${ }^{4,5}$. The commonly used grading system by Matta/Tournetta ${ }^{6}$ does not fulfil any of these criteria.

More important, the true impact of pelvic ring fracture reduction has not been investigated. Perfect reduction often requires greater surgical approaches and longer operating times, which also may negatively influence a patient's outcome. Information on the correlation between grade of reduction and these patients' outcome is therefore important.

This retrospective analysis aimed to investigate the correlation of functional outcome in patients with pelvic fractures as measured by the Majeed Score and Time Up \& Go Test with the postoperative radiographic quality of reduction measured by the three techniques as described by Matta/Tournetta, Sagi and Keshishyan. 


\section{Patients and Methods}

This study was carried out in accordance with the local institutional ethics committee's terms of reference (Kantonale Ethikkommission Zürich, Switzerland. KEK-ZH-Nr. 2017-01232).

\section{Patients}

From a prospective pelvic fracture database, consecutive patients $(n=68)$ with a traumatic pelvic ring fracture not involving the acetabulum that required closed or open reduction and fixation between 07/2015 and 07/2017 were identified. Further inclusion criteria were union of the fracture at 6 months follow-up, the presence of an informed consent and a complete functional follow-up at 6 months and at last follow-up. Fracture union was defined as anterior and posterior cortical bridging of the pelvic

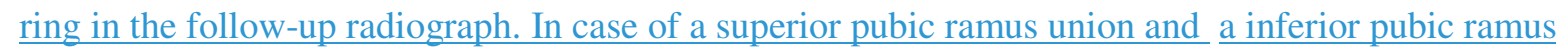
non-union, this counted as "union" of the anterior pelvic ring. Patients with other injuries to the lower extremities $(n=16)$, declined informed consent $(n=4)$ and incomplete follow up $(n=17)$ were excluded.

This left 31 patients (mean age 50 years, SD 21 years, range, 16 to 88 years; 17 female) for final evaluation. Outcome data was obtained from the same prospective database and in addition a retrospective chart review was performed. Postoperative quality of pelvic ring reduction was determined by three established methods as described below.

\section{Radiographic quality of reduction}

The quality of pelvic ring reduction was quantified on postoperative radiographs by three established methods as described by Matta/Tournetta ${ }^{6}$, Sagi ${ }^{7}$, and Keshishian/Lefaivre ${ }^{4,5}$.

Conventional digitalized antero-posterior radiographs of the pelvis were taken using a MULTIX / Optitop 150/40/80 tube (Siemens, Munich, Germany; 71-90 kV, 25-40 mA). The radiographs were all taken in a standardized fashion with a film-focus distance of $115 \mathrm{~cm}$. Radiographic measurements were performed by the use of standard caliper tools provided by the institutional imaging software (AGFA® Impax viewer, Mortsel, Belgium). 
The method described by Matta and Tournetta ${ }^{6}$ estimates the grade of reduction by rating without taking measurements. The examiner's impression of reduction is then assigned to one of four groups: excellent $(0-4 \mathrm{~mm})$, good $(4-10 \mathrm{~mm})$, fair $(10-20 \mathrm{~mm})$ and poor $(>20 \mathrm{~mm})$. This method was applied and results were documented before actual measurements for the two other techniques were done in order to avoid a bias.

\section{The inlet/outlet ratio as described by Sagi ${ }^{7}$}

This method uses measurements of inlet and outlet views only. A line perpendicular to a reference line through the spinal processes is drawn at the anterior border of the Sacrum in inlet views and superior boarder of S1 in outlet views. Next the perpendicular distance of the subchondral bone of each acetabulum to the line is measured (Figure 1). The Sagi-score is a simple ratio of these distances using the effected side as the numerator

\section{The cross measurement technique as initially described by Keshishyan and modified by Lefaivre ${ }^{4,5}$}

This method was first described by Keshishyan ${ }^{5}$ pelvic displacement in pediatric patients and uses standard a.p. pelvic views only. A line is drawn on each side from the inferior sacroiliac joint (iliac side) to the contra lateral triradiate cartilage in skeletal immature patients. Lefaivre ${ }^{4}$ modified this technique in adults by talking measurements to the contra lateral teardrop, a reliable radiologic marker which was defined as the most distal radio dense area below the acetabulum and is located in the anteroinferior portion of the acetabular fossa at the acetabular notch ${ }^{8}$ " $\mathrm{X}$ " is the distance from the right sacroiliac joint to the left teardrop; "Y" is the opposite of this (Figure 2). This allows for three possible interpretations. 1 . The pelvic asymmetry value ABS (X-Y). 2. The pelvic deformity index [ABS $(\mathrm{X}-\mathrm{Y}) /(\mathrm{X}+\mathrm{Y})]$ which considers for projection errors. 3. The simple Ratio $(\mathrm{X} / \mathrm{Y})$ as described by Lefaivre ${ }^{4}$.

All radiographic measurements were performed by the first author. Each measurement was repeated three times for each single distance of interest and then averaged to reduce the margin of error. The intra-observer reliability of radiographic measurements was calculated. 


\section{Functional outcome measurement}

All patients included into this study from a prospective outcome database had a completed Majeed and Timed Up \& Go at 6 months and at last follow-up (mean 10 months, SD 5 months, range, 6 to 24 months).

The Majeed score consists of seven items (pain, work, sitting, sexual intercourse, walking aids, gait unaided, walking distance) and has a maximum of 100 points. As some patients did not work prior to the trauma and some did not have sexual intercourse within the year prior to the trauma the achievable maximum value varied among the included patients. To correct for this, final analysis was made using the percentage of achievable maximum Majeed score (e.g. 65 of 80 points $=81.3 \%$ in a patient who did not work before the accident).

The Timed Up \& Go measures the time taken to stand up, walk a distance of $3 \mathrm{~m}$, turn around, walk back and sit down on the chair again ${ }^{9}$. It has originally been described for geriatric patients to predict falls and function in patients with musculoskeletal conditions.

\section{Statistical Analysis}

Statistical analysis was done using SPSS for Windows 23.0 (SPSS, Chicago, Illinois, USA). Data are presented as means (continuous data) and standard deviation (SD).

Correlations between continuous data sets were tested using the Pearson's correlation coefficient after graphic confirmation of a normal distribution. The level of statistical significance was set at $\mathrm{p} \leq 0.05$. For intra-observer reliability of the radiographic measurements, intra-class correlation coefficients (ICC 1,1) and 95\% CIs were calculated. 


\section{Results}

Thirty-one patients (mean age 50 years, SD 21 years; 17 female) with an isolated pelvic fracture of different severity (Young \& Burgess LC1, LC2, LC 3, APC2, VS) that required closed or open reduction and fixation were available for final analysis. Fracture reduction and fixation was performed by open techniques through an anterior approach (Stoppa or ilio-inguinal) in 22 cases and by solely closed percutaneous techniques_i.e. with Schanz pins and/or external fixators and traction/ compression/distraction) in nine cases. Seven patients had sustained bilateral posterior injuries to the pelvis.

\section{$\underline{\text { Two patients had concomitant uro-genital injuries. One patient had a bladder perforation from a sharp }}$} bone fragment and one had a skin laceration close to the penis insertion with a perineal hematoma. In both cases, the functional outcome as assessed by Majeed or Timed Up\&Go at 6 months after the trauma was not affected by these injuries. The mean Injury Severity Score was 20.6, SD 11.6.

\section{Radiographic quality of reduction}

Postoperative quality of pelvic ring reduction was graded according to Matta (median 2.0, range 1 to 3), the Sagi inlet/outlet ration (mean 0.99 , range 0.75 to 1.36 ), and as described by Keshishian/Lefaivre (mean pelvic asymmetry 11.4, SD 8.4; mean pelvic deformity index 0.041 , SD $0.030)$.

For all methods, the intra-observer reliability was high (ICC 1.1). Using the measurement according to Keshishian yielded a reliability of 0.996 (95\% CI, 0.993 to 0.998) for the "X" diameter and 0.989 (95\% CI, 0.981 to 0.949 ) for "Y" diameter. The method described by Sagi for inlet views of the pelvis showed a reliability of 0.966 (95\% CI, 0.940 to 0.982$)$ for the right side and 0.945 (95\% CI, 0.903 to 0.971) for the left side. For outlet views of the pelvis the reliability was 0.969 (95\% CI, 0.945 to 0.984) for the right side and 0.976 (95\% CI, 0.958 to 0.988$)$ for the left side.

\section{Functional outcome measurement}

The mean Majeed score was 78, SD 16 at 6 months (89.2\% of achievable maximum) and 78, SD 18 (88.0 \% of achievable maximum) at last follow-up. All patients were able to perform the Timed Up \& 
166 Go test; the mean duration was $10 \mathrm{sec}, \mathrm{SD} 10 \mathrm{sec}$ at 6 months and $10 \mathrm{sec}$, SD $14 \mathrm{sec}$ at last follow-up.

167 One Patient needed walking aids at 6 months follow up.

\section{Correlation of quality of reduction and functional outcome}

170 The functional outcome at 6 months as measured by Majeed correlated moderately with the pelvic asymmetry value (Pearson R: $-0.520, \mathrm{p}=0.003)$ and the pelvic deformity index $(-0.527, \mathrm{p}=0.002)$ as

172 described by Keshishian/Lefaivre. This correlation decreased with time (last follow-up: -0.302,

$173 \mathrm{p}=0.099 /-0.322, \mathrm{p}=0.077)$.

174 The functional outcome at 6 months as measured by the Timed Up \& Go showed a similar correlation

175 with the pelvic asymmetry value $(0.585, \mathrm{p}=0.001)$ and the pelvic deformity index $(0.503, \mathrm{p}=0.004)$. As

176 seen for the Majeed this association decreased with time (last follow-up: 0.259, p=0.160 / 0.297, $177 \mathrm{p}=0.104)$.

178 There was a weak correlation between the Timed Up \& Go and the radiographic grading system as 179 described by Matta/Tournetta at 6 months $(0.408, \mathrm{p}=0.023)$ and last follow-up $(0.380, \mathrm{p}=0.035)$.

180 No correlation was found between the Majeed and the radiographic grading system as described by

181 Matta/Tournetta at 6 months $(-0.279, \mathrm{p}=0.129)$ and last follow-up $(-0.058, \mathrm{p}=0.755)$.

182 Neither a significant correlation between the Majeed at 6 months $(0.356, \mathrm{p}=0.172)$ and last follow-up 183 (0.251, p=0.213) nor of the Timed Up \& Go at 6 months $(0.168, \mathrm{p}=-0.254)$ and last follow-up (0312, $\mathrm{p}=0.188$ ) with the system described by Sagi could be found. 


\section{Discussion}

The purpose of our study was to correlate the functional outcome in patients with pelvic fractures with the postoperative radiographic quality of reduction.

Functional outcome was assessed by the outcome instrument most commonly used in studies on pelvic fractures, the Majeed score. In addition, the Timed Up \& Go test was used as a measure for walking ability and velocity. Three techniques were chosen to measure radiographic deformity: The one most commonly used in the literature (Matta/Tournetta) and the two with the best inter- and intra-observer reliability (Sagi, Keshishian/Lefaivre) ${ }^{4-7}$.

While there was no correlation found between the functional outcome scores and the radiographic grading system as described by Sagi, we observed a moderate correlation of both clinical outcome scores at 6 months with postoperative quality of radiographic reduction when measured with the method described by Keshishyan/Lefaivre. Only a weak correlation was observed between the Timed Up \& Go and the radiographic grading system as described by Matta/Tournetta.

The importance of anatomic reconstruction of the pelvic ring has been stressed, but other factors like primary neurological/urological injuries can have a relevant impact, too ${ }^{6,10}$. For this study, only patients with a healed fracture were included in order to focus on the sequelae of deformity rather than non-union or infection. Only two patients in our cohort had uro-genital injuries, both not affecting functional outcome, in particular sexual intercourse. Hence, the results may only be applied to patients without complications other than malunion.

The number of patients included is rather small $(n=31)$ and may not represent a comprehensive picture of potential deformities. We did not differentiate by rotational or vertical displacement, nor was the sample big enough to do subgroup analyses for patients with uni- vs. bilateral injuries.

However, the radiographic measurement techniques used had very different approaches in determining "pelvic deformity". Maybe, assessing the whole pelvic ring's symmetry does make more sense thatn only looking at pure displacement: The cross measurement technique method by Keshishyan is associated with a better interobserver reliability ${ }^{4}$ and showed a better correlation with functional outcome in our study. The grading system by Matta and Tournetta, in contrast, focuses on 
212 displacement and is difficult to reproduce and, thus, has shown to have poor interobserver reliability

213 and a much weaker correlation with function.

214 With a complete follow-up of 6 months for all patients and a last follow-up of mean 10 months, it is

215 well conceivable that further functional improvement may have occurred beyond this study's

216 observations. Even though no functional improvement was seen between the follow-up at 6 months

217 (Majeed 78 points) and the last follow-up (Majeed 78 points) in our cohort, future studies with longer

218 follow-ups need to confirm the findings of this study.

219 The functional outcome scores used in this study are susceptible for ceiling effects. Therefore the

220 ability to differentiate the outcome in young and otherwise healthy patients may be effected. In

221 addition, the item "working" of the Majeed score is sometimes difficult to assess and may be affected

222 by the upper extremities' function, as well. However, there exists no well validated score for

223 functional outcome after pelvic ring injuries ${ }^{11}$. The Majeed score is the the one by most studies on this

224 topic. And while the Timed Up \& Go had has originally been described for geriatric patients

225 (Podsiadlo und Richardson 1991), it can be used to assess impairments of activities of daily living that

may potentially be affected by pelvic deformity.

Functional results after pelvic trauma are often affected by other associated injuries and other variables ${ }^{12}$. For this reason, patients with injuries to the lower extremities were excluded in this study due to the potential effect on functional outcome scores. In severe pelvic trauma a high incidence of these injuries is reported ${ }^{1}$. This may affect the external validity of our results.

Although having only descriptive value our findings underline the importance of anatomic reduction and restoration of pelvic symmetry in patients with pelvic trauma. More invasive surgical approaches and longer operating times may sometimes be necessary to avoid gross malalignment. The cross measurement technique as described by Keshishyan/Lefaivre correlated best with functional outcome and showed high intraobserver reliability. This could support its value as a prognostic tool in patients with pelvic fractures.

Future studies with more patients and more investigators are required to confirm our findings. and Rreliability and validity of functional outcome scores needs to be further assessed to predict outcome in patient with fractures of the pelvic ring. 
241 Conclusions

242 Based on the results of this study the cross measurement technique as described by

243 Keshishyan/Lefaivre shows a moderate correlation to functional outcome scores and could be a

244 valuable outcome predictor in patients with pelvic ring fractures with high intra- and interobserver 245 reliability.

246

247 Acknowledgements

248 There were no external sources of funding for this study. 


\section{References}

1. Osterhoff G, Scheyerer MJ, Fritz Y, Bouaicha S, Wanner GA, Simmen HP et al. Comparing the predictive value of the pelvic ring injury classification systems by Tile and by Young and Burgess. Injury 2014;45(4):742-7.

2. Buller LT, Best MJ, Quinnan SM. A Nationwide Analysis of Pelvic Ring Fractures: Incidence and Trends in Treatment, Length of Stay, and Mortality. Geriatr Orthop Surg Rehabil 2016;7(1):9-17.

3. Tornetta, P., 3rd, Matta JM. Outcome of operatively treated unstable posterior pelvic ring disruptions. Clinical orthopaedics and related research 1996(329):186-93.

4. Lefaivre KA, Blachut PA, Starr AJ, Slobogean GP, O'Brien PJ. Radiographic displacement in pelvic ring disruption: reliability of 3 previously described measurement techniques. Journal of orthopaedic trauma 2014;28(3):160-6.

5. Keshishyan RA, Rozinov VM, Malakhov OA, Kuznetsov LE, Strunin EG, Chogovadze GA et al. Pelvic polyfractures in children. Radiographic diagnosis and treatment. Clinical orthopaedics and related research 1995(320):28-33.

6. Matta JM, Tornetta, P., 3rd. Internal fixation of unstable pelvic ring injuries. Clinical orthopaedics and related research 1996(329):129-40.

7. Sagi HC, Militano U, Caron T, Lindvall E. A comprehensive analysis with minimum 1-year follow-up of vertically unstable transforaminal sacral fractures treated with triangular osteosynthesis. Journal of orthopaedic trauma 2009;23(5):313-9; discussion 319-21.

8. Bowerman JW, Sena JM, Chang R. The teardrop shadow of the pelvis; anatomy and clinical significance. Radiology 1982;143(3):659-62.

9. Podsiadlo D, Richardson S. The timed "Up \& Go": a test of basic functional mobility for frail elderly persons. J Am Geriatr Soc 1991;39(2):142-8.

10. Pohlemann T, Gansslen A, Schellwald O, Culemann U, Tscherne H. [Outcome evaluation after unstable injuries of the pelvic ring]. Der Unfallchirurg 1996;99(4):249-59.

11. Lefaivre KA, Slobogean GP, Valeriote J, O'Brien PJ, Macadam SA. Reporting and interpretation of the functional outcomes after the surgical treatment of disruptions of the pelvic ring: a systematic review. The Journal of bone and joint surgery. British volume 2012;94(4):549-55. 
Quality of reduction and outcome in pelvic fractures

277 12. Hessmann MH, Rickert M, Hofmann A, Rommens PM, Buhl M. Outcome in Pelvic Ring 278 Fractures. Eur J Trauma Emerg Surg 2010;36(2):124-30.

279 


\section{Figure legends}

\section{Figure 1 The inlet/outlet ratio}

283

284

285

286

287

288

289

290

291

292

293

294

295

On inlet and outlet views of the pelvis, the right and left orthogonal distances (lines X and Y) from the acetabular dome to a (dotted) reference line are determined. A ratio is calculated with the injured side being the numerator. The reference line in the inlet view runs tangentially along the anterior sacral wall towards (and rectangular to the spinal processes). In the outlet view, it runs parallely to the upper endplate of S1 (rectangular to the spinal processes).

\section{Figure 2 The cross measurement technique}

On AP radiographs of the pelvis, a line is drawn connecting each inferior sacroiliac joint and the contralateral Köhler's teardrop. «Y» is the distance from the left sacroiliac joint to the right Köhler's teardrop and «X» is the distance from the right sacroiliac joint to the left Köhler's teardrop. This allows for three interpretations: 1 . The pelvic asymmetry value being the difference $(X-Y), 2$. the deformity index [ABSOLUTE $(\mathrm{X} 2 \mathrm{Y}) / \mathrm{X}+\mathrm{Y}]$, and 3. the simple ratio $(\mathrm{X} / \mathrm{Y})$. 